\title{
Pequena central hidrelétrica afeta a estrutura populacional do lambari do rabo vermelho Psalidodon aff. fasciatus (Cuvier 1819)
}

\author{
Small hydroelectric power plant affects the population structure of lambari of the red tail \\ Psalidodon aff. fasciatus (Cuvier 1819) \\ Pequeña central hidroeléctrica afecta la estructura poblacional del lambari cola roja Psalidodon \\ aff. fasciatus (Cuvier 1819)
}

Recebido: 22/07/2021 | Revisado: 27/07/2021 | Aceito: 29/07/2021 | Publicado: 05/08/2021

Rogério Anderson Druzian

ORCID: https://orcid.org/0000-0002-8421-6874

Universidade Estadual do Oeste do Paraná, Brasil

E-mail: druzian.ra@gmail.com

José Rafael Soares Fonseca

ORCID: https://orcid.org/0000-0003-1934-333X

Universidade Estadual do Oeste do Paraná, Brasil E-mail: ra.phb@hotmail.com

Jean Colombari Neto

ORCID: https://orcid.org/0000-0002-0744-6297

Universidade Estadual do Oeste do Paraná, Brasil

E-mail: jean.c_neto@hotmail.com

Tiago Debona

ORCID: https://orcid.org/0000-0002-6084-7241

Instituto Neotropical de Pesquisas Ambientais, Brasil

E-mail: tiago.debona@gmail.com

Vinicius Valiente dos Santos

ORCID: https://orcid.org/0000-0001-8388-1955

Instituto Neotropical de Pesquisas Ambientais, Brasil

E-mail: viniciusvaliente@hotmail.com

Pedro Rogério Leandro da Silva

ORCID: https://orcid.org/0000-0002-1003-1594

Instituto Neotropical de Pesquisas Ambientais, Brasil

E-mail: pedropesca@yahoo.com.br

Anderson Luís Maciel

ORCID: https://orcid.org/0000-0002-3124-7271

Instituto Neotropical de Pesquisas Ambientais, Brasil

E-mail: maciel_ander@yahoo.com.br

Carlos Henrique Orsi

ORCID: https://orcid.org/0000-0002-1837-6757

Instituto Neotropical de Pesquisas Ambientais, Brasil

E-mail: carlos.engpesca@gmail.com

Cleomar Fernandes

ORCID: https://orcid.org/0000-0003-0825-8236

Instituto Neotropical de Pesquisas Ambientais, Brasil

E-mail: cleomar_pesca@hotmail.com

Antonio Nogueira dos Reis

ORCID: https://orcid.org/0000-0002-1071-8847

Instituto Neotropical de Pesquisas Ambientais, Brasil

antonio.nogueira@hotmail.com

Cleodimar Fernandes

ORCID: https://orcid.org/0000-0001-8603-7872

Instituto Neotropical de Pesquisas Ambientais, Brasil

E-mail: cleodimarf@hotmail.com

Gilmar Baumgartner

ORCID: https://orcid.org/0000-0003-0912-482X

Universidade Estadual do Oeste do Paraná, Brasil

E-mail: gilmarbaum03@gmail.com

\section{Resumo}

Recentemente no Brasil começaram a ser construídas pequenas centrais hidrelétricas em rios de pequena ordem para aumentar a capacidade energética, e com isso, o rio São Francisco Verdadeiro foi barrado para a formação do reservatório da PCH São Francisco. O objetivo deste trabalho foi verificar se a formação do reservatório da PCH São 
Francisco, alterou positivamente a estrutura populacional de Psalidodon aff. fasciatus. As coletas trimestrais dos peixes foram realizadas em três locais amostrais, com redes de espera, nas fases pré e pós enchimento. Foram verificadas diferenças significativas na abundância, nos locais do reservatório e jusante. Na estrutura em tamanho, observou-se maior frequência de indivíduos de menor tamanho a jusante e no reservatório. Com relação à atividade reprodutiva, houve redução da intensidade no reservatório no período imediatamente após o represamento. Quanto ao fator de condição no reservatório e na jusante foram verificadas alterações positivas. Deste modo, concluímos que a implantação da PCH São Francisco influenciou positivamente a abundância, estrutura em tamanho e fator de condição de P. aff. fasciatus, o que está relacionado à maior disponibilidade de alimento e habitats após o barramento.

Palavras-chave: Atividade reprodutiva; Captura; Fator de condição; Ictiofauna.

\begin{abstract}
Recently in Brazil began to be built small hydroelectric plants on small rivers order to increase energy capacity. The São Francisco real river was barred to form the reservoir of PCH São Francisco. The aim of this work was to verify if the reservoir of PCH São Francisco, changed the population structure of Psalidodon aff. fasciatus. Quarterly samples of fish were held at three sampling sites, with gillnets, in phases before and after the filling of the reservoir. Significant differences were observed in the abundance in the reservoir and downstream sites. In size structure, higher frequency of smaller individuals downstream and into the reservoir. With respect to reproductive activity, there was a reduction of the intensity in the reservoir in the period immediately after the construction of the dam. As for the condition factor in the reservoir and downstream were observed positive changes. Therefore, we conclude that the PCH São Francisco influenced positively the abundance and size structure and the condition factor of $P$. aff. fasciatus, which is related to the greater availability of food and habitats after the construction of the dam.
\end{abstract}

Keywords: Condition factor; Reproductive activity; Catch; Ichthyofauna.

\title{
Resumen
}

Recientemente, en Brasil, se comenzaron a construir pequeñas centrales hidroeléctricas en pequeños ríos para aumentar la capacidad energética, y con eso, el río São Francisco Verdadeiro fue represado para la formación del embalse de la PCH São Francisco. El objetivo de este trabajo fue verificar si la formación del embalse de la PCH São Francisco alteró positivamente la estructura poblacional de Psalidodon aff. fasciatus. Las colecciones trimestrales de pescado se realizaron en tres lugares de muestreo, con redes de espera, en las fases de pre y posllenado. Se encontraron diferencias significativas em la abundancia en el embalse y en los sitios río abajo. En la estructura en tamaño, fue observado una mayor frecuencia de individuos más pequeños em aguas abajo y en el embalse. En cuanto a la actividad reproductiva, hubo una reducción de la intensidad en el embalse en el período inmediatamente posterior al embalse. En cuanto al factor de condición en el embalse y aguas abajo, se verificaron cambios positivos. Por lo tanto, concluimos que la implementación del PCH São Francisco influyó positivamente en la abundancia, estructura de tamaño y factor de condición de $P$. aff. fasciatus, lo que se relaciona con la mayor disponibilidad de alimentos y hábitats después de la represa.

Palabras clave: Actividad reproductiva; Captura; Factor de condición; Ictiofauna.

\section{Introdução}

A construção de usinas hidrelétricas gerando barramentos em rios é uma ação antrópica que causa impactos aos ecossistemas aquáticos (Mérona \& Albert, 1999; Agostinho et al., 2007; Araújo et al., 2013; Normando et al., 2014; Novaes et al., 2014; Holcomb et al., 2015; Affonso et al., 2015; Poff e Schmidt 2016; Oliveira et al., 2018). Com o enchimento do reservatório, os habitats que existiam anteriormente são alterados, sendo alguns extintos, como por exemplo, corredeiras, poços, lagoas marginais e canais, enquanto novos habitats podem ser formados, como bancos de areia, zonas de macrófitas e galhadas (Agostinho et al., 2007; Ferreira, 2020). A montante da barragem a velocidade da água é amplamente alterada, deixando de ter fluxo lótico, se tornando um ambiente lêntico (Bazzoli et al.,1991; Lowe-Mcconnell, 1999; Agostinho et al., 2007; Agostinho et al., 2016). A jusante do barramento o antigo rio sofre mudanças, principalmente devido à alteração na vazão natural de água (Cooper et al., 2016), além disso, as espécies de peixes migradores não conseguem completar seu ciclo migratório, sendo impedidas pela barragem de alcançar as cabeceiras dos rios e não se reproduzem (Agostinho et al., 2007; 2016; Cooper et al., 2016).

Após o barramento existe uma tendência de reestruturação das espécies de peixes no reservatório (Agostinho et al., 2007), algumas espécies podem ser prejudicadas, enquanto outras se beneficiam. Entre as espécies que podem ser beneficiadas estão aquelas que possuem desova parcelada, desovando várias vezes dentro do mesmo ciclo reprodutivo (Vazzoler 1996; 
Lowe-Mcconnell, 1999), ou que apresentam ciclo de vida precoce, em que os recrutas rapidamente se tornam aptos à reprodução (Vazzoler, 1996). Essas características são apresentadas principalmente por peixes de pequeno porte (Agostinho et al., 2016), entre eles os lambaris do gênero Astyanax.

O rio São Francisco Verdadeiro foi barrado para a formação do reservatório da PCH São Francisco, neste rio, a maioria das espécies de peixes são de pequeno porte, dentre elas destaca-se $P$. aff. fasciatus. Esta espécie, vulgarmente conhecida como lambari do rabo vermelho é pertencente à família Characidae e possui ampla distribuição, sendo encontrada em bacias hidrográficas da América do Sul e América do Norte (Graça \& Pavanelli, 2007), comumente habita lagoas e rios e apresenta preferência alimentar por detritos em águas de fluxo lótico, vegetais e larvas de insetos em fluxo lêntico, aproveitando-se da planície de inundação nas cheias (Hahn et al., 2004). Anteriormente seu nome cientifico era Astyanax fasciatus, mas, uma revisão taxonômica reintroduziu o gênero Psalidodon (Terán et al. 2020). Sua biologia reprodutiva é característica de espécie de migração de curta distância, apresenta primeira maturação precoce (4,7 cm para fêmeas e machos), se reproduz por amplo período entre agosto e fevereiro sem cuidado parental (Suzuki et al., 2004; Graça \& Pavanelli, 2007), características típicas de espécies estrategistas oportunistas (Winemiller, 1989, Viana et al. 2020).

Considerando as características desta espécie, este trabalho teve como objetivo verificar se a formação do reservatório PCH São Francisco, alterou a estrutura populacional de P. aff. fasciatus. Deste modo, as hipóteses aqui testadas foram de que a formação do reservatório influenciou positivamente na: I) abundância, II) estrutura em tamanho, III) atividade reprodutiva, e IV) fator de condição desta espécie.

\section{Metodologia}

O rio São Francisco Verdadeiro, localizado inteiramente no estado do Paraná, nasce no município de Cascavel e desemboca no reservatório de Itaipu, no município de Entre Rios do Oeste. A sua área de drenagem é de 2.219,1 km², abrange os municípios de Cascavel, Toledo, Ouro Verde do Oeste, São José das Palmeiras, Entre Rios do Oeste, Marechal Cândido Rondon e Pato Bragado (Gubiani et al., 2010).

A pequena central hidrelétrica PCH São Francisco está localizada entre os municípios de Toledo e Ouro Verde do Oeste (latitude $24^{\circ} 43^{\prime} 56,72^{\prime \prime}$ e longitude de $53^{\circ} 53^{\prime} 32,81^{\prime \prime}$ ) e conta com um reservatório com extensão aproximada de 4 km, a profundida máxima do reservatório é aproximadamente 20 metros. A tomada de água para as duas unidades geradoras é através de dois tubos de adução, que captam água da superfície até 5 metros de profundidade, e destina a água pela tubulação por 70 metros de desnível, até alcançar a duas turbinas tipo Francis, com potência total de 14,0 MW.

Para testar as hipóteses e alcançar os objetivos, foram estabelecidos três sítios de amostragem na área de influência da

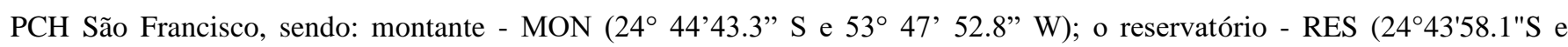

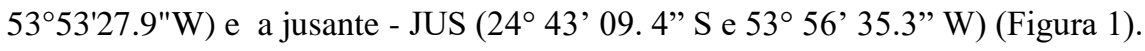


Figura 1. Localização dos locais de amostragem na área de influência da PCH São Francisco, rio São Francisco Verdadeiro.
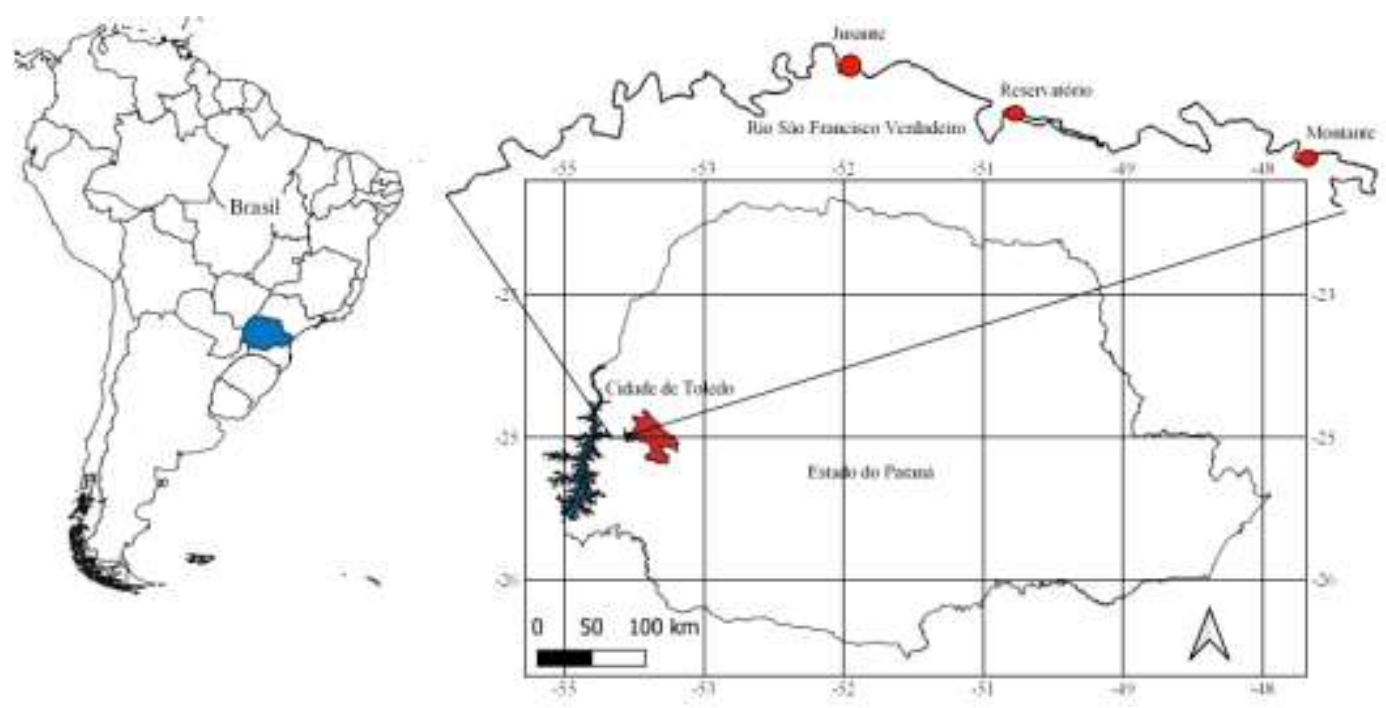

Fonte: Autores.

As coletas foram realizadas trimestralmente pelo Grupo de Pesquisa em Recursos Pesqueiros e Limnologia (GERPEL), da Universidade Estadual do Oeste do Paraná (Unioeste), entre dezembro de 2007 e setembro de 2010 , considerada a fase de pré-enchimento do reservatório (Pré), dezembro de 2010 e setembro de 2013, denominada primeira fase pós-enchimento (Pós I) e de dezembro de 2013 a setembro de 2016, denominada segunda fase pós-enchimento (Pós II). Em todos os três períodos o tempo de amostragem foi similar com 36 meses e 12 coletas cada.

Para a captura na fase Pré foram utilizadas redes de malhar simples $(2,5 ; 3,0 ; 4,0$ e 5,0 cm de entre nós não adjacentes), todas com 10 metros de comprimento, expostas nas margens (Figura 2 A).

Nas fases pós-enchimento foi incluído um conjunto de redes no local do reservatório, exposto na superfície (Figura 2 B). As redes foram instaladas às 16 horas e retiradas na manhã seguinte, revistadas às 22:00 e 08:00 horas, ficando expostas por aproximadamente 16 horas. Este protocolo de amostragem foi aprovado pelo Comitê de Ética em experimentação Animal da Unioeste.

Figura 2. A - Revista e retirada de rede de espera com 10 metros de comprimento exposta na margem. B - Bateria de rede de superfície exposta no reservatório da PCH São Francisco Verdadeiro.
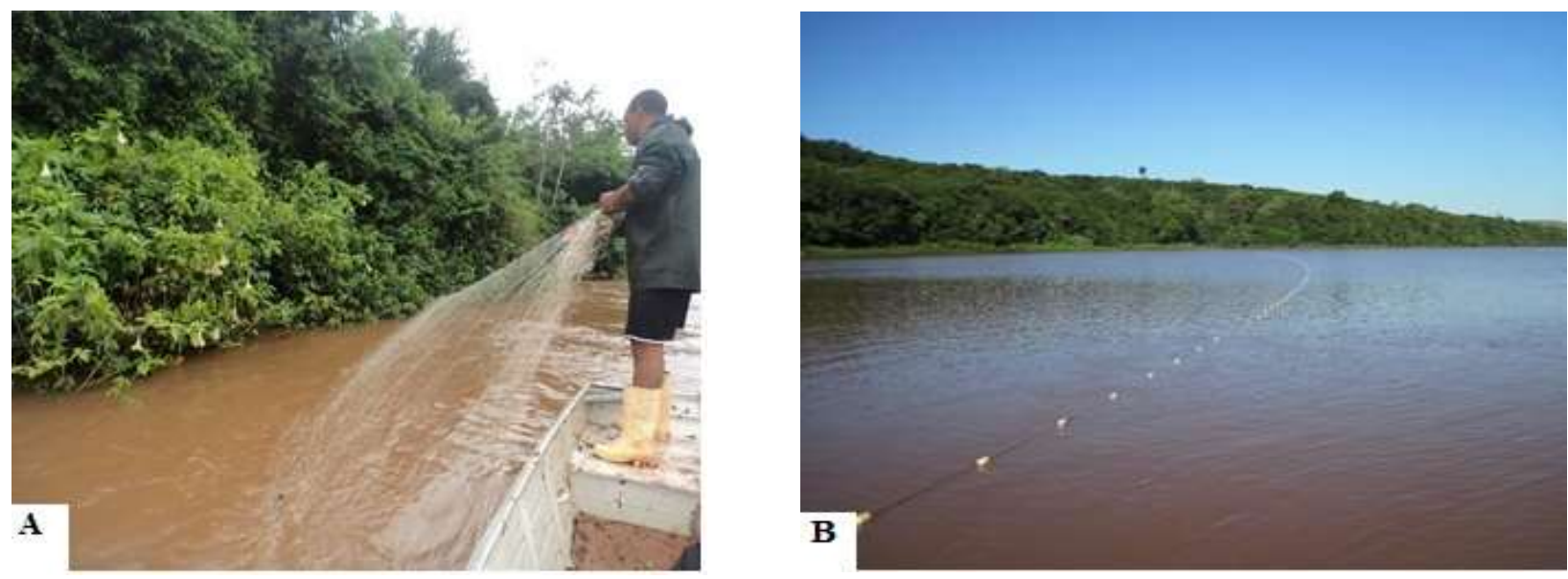

Fonte: Autores. 
Os peixes depois de capturados foram eutanasiados em solução de Benzocaína (250mg/L) (Okamura et al., 2010) e conservados em gelo até a análise. A identificação foi efetuada segundo Graça \& Pavanelli (2007) e exemplares testemunhos foram depositados na coleção ictiológica do Gerpel.

De todos os exemplares foram obtidos: comprimento total (Lt), comprimento padrão (Ls), peso total (Wt), peso das gônadas (Wg), sexo e estádio de desenvolvimento gonadal. A determinação dos sexos e dos estádios de desenvolvimento gonadal foi efetuada através da inspeção macroscópica das gônadas, observando-se o tamanho, a forma e a possibilidade de visualização dos ovócitos, conforme metodologia proposta por Vazzoler (1981 e 1996).

A abundância foi determinada através da captura por unidade de esforço (CPUE) para cada amostra obtida segundo King (2005). Para o cálculo foi considerado o número de indivíduos capturados (CPUEn) e o peso total capturado (CPUEb), sendo o esforço dado para cada 1000 metros quadrados de rede em 16 horas de exposição. Assim tem-se: CPUE =C/E * 1000, onde: CPUEn, $\mathrm{b}=$ número de indivíduos (ou biomassa) capturados por $1000 \mathrm{~m}^{2}$ de redes*16h; $\mathrm{C}=$ número de exemplares (ou biomassa) e $\mathrm{E}=$ esforço de pesca utilizado ( $\mathrm{em} \mathrm{m}^{2}$ de rede*16h).

Para estabelecer a estrutura em tamanho, a amplitude de variação de comprimento foi dividida em 11 classes, segundo fórmula de Sturges (Sokal \& Rohlf, 1981). O cálculo da porcentagem de indivíduos por classe foi realizado, dividindo o número de fêmeas capturadas pertencente a classe i, pelo número total de fêmeas capturadas e o resultado multiplicado por 100 .

A intensidade reprodutiva foi avaliada através do índice de atividade reprodutiva (IAR), adaptado de Vazzoler (1996) e Baumgartner et al. (2016), para as fêmeas. Assim tem- se:

$$
\mathrm{IAR}=\frac{\ln C P U E n N i\left(\frac{n i}{\sum n i}+\frac{n i}{C P U E n N i}\right) \frac{R G S i}{R G S e}}{\ln N m\left(\frac{n m}{\sum n i}+1\right)} * 100
$$

Onde: CPUEn $=$ CPUEn na unidade amostral $\mathbf{i} ; \mathrm{ni}=\mathrm{n}^{\circ}$ de fêmeas em reprodução na unidade amostral $\mathbf{i} ; \mathrm{Nm}=\mathrm{n}^{\circ}$ de fêmeas na maior unidade amostral; $\mathrm{nm}=\mathrm{n}^{\circ}$ de fêmeas em reprodução na unidade amostral com maior $\mathbf{n}$; RGSi $=$ RGS médio das fêmeas em reprodução na unidade amostral i; RGSe = maior valor individual da RGS; RGS= peso dos ovários*100/peso total.

A atividade reprodutiva foi considerada nula (IAR $\leq 2)$, incipiente $(2<$ IAR $\leq 5)$, moderada $(5<$ IAR $\leq 10)$, intensa $(10<$ IAR $\leq 20)$ e muito intensa (IAR > 20), segundo Vazzoler (1996).

Como o índice de atividade reprodutiva (IAR), necessita um número mínimo de indivíduos (Suzuki et al., 1997; Agostinho et al., 1997), foi necessário agrupar os dados por ano, ao invés de trimestre, tendo sido utilizadas apenas as fêmeas, como empregado por Agostinho et al. (1991) e Baumgartner et al. (2016).

A higidez, foi avaliada para fêmeas, através do fator de condição total, segundo Vazzoler (1996), utilizando o cálculo: $\mathrm{K}=\mathrm{W}^{\mathrm{t}} / \mathrm{L}_{\mathrm{t}}^{\mathrm{b}}$, onde: $\mathrm{K}=$ fator de condição total; $\mathrm{Wt}=$ peso total; $\mathrm{Lt}=$ comprimento total; $\mathrm{b}=$ coeficiente angular da regressão $\mathrm{Wt} / \mathrm{Lt}$.

Os resultados foram submetidos à checagem dos pressupostos utilizando o teste de Levine e Shapiro-Wilk, e no caso da CPUE que não atendeu a estes pressupostos os dados foram transformados em Raiz Quadrada. Para a verificação de diferenças significativas, os dados foram comparados utilizando-se a Análise de Variância Unifatorial (One-Way Anova) e quando encontradas diferenças significativas foi aplicado o teste de Tukey (Zar, 1999). Todos os testes foram realizados ao nível de 5\% de significância, utilizado o software Statistica 7.1. 


\section{Resultados}

No local montante não foram verificadas diferenças significativas ( $\mathrm{p}>0,05)$ para biomassa média capturada, sendo que no primeiro período pós-enchimento (Pós I) houve redução na abundância. A mesma tendência de redução pôde ser observada para a CPUEn que também não houve diferença significativa ( $p>0,05)$ (Tabela 1). Porém, no reservatório foram verificadas diferenças significativas $(\mathrm{p}<0,05)$ tanto para CPUEn, como para CPUEb, o que caracteriza um aumento na abundância média capturada, tanto para biomassa quanto para número de indivíduos na segunda fase (Pós I) após o enchimento do reservatório. Foi registrada no período Pré uma CPUEb de $2,23 \pm 0,25 \mathrm{~kg} / 1000 \mathrm{~m}^{2}$ de redes*16h, enquanto no período Pós II foi de $3,19 \pm$ $0,22 \mathrm{~kg} / 1000 \mathrm{~m}^{2}$ de redes*16h. Para CPUEn as médias foram de 15,77 $\pm 1,70$ indivíduos/1000m² de redes*16h no período Pré e de 29,38 \pm 2,47 indivíduos/1000m² de redes*16h no período Pós II (Tabela 1 ).

$\mathrm{Na}$ jusante também foram verificadas diferenças significativas na abundância $(\mathrm{p}<0,05)$, tanto para a CPUEn como para CPUEb, sendo que para ambas os maiores valores foram registrados após o enchimento do reservatório. Para CPUEn no período Pré foi registrado o menor valor 2,37 $\pm 1,06$ indivíduos/1000m² de redes*16h e no período Pós I $17,39 \pm 3,53$ indivíduos $/ 1000 \mathrm{~m}^{2}$ de redes*16h. Para a CPUEb o menor valor foi também registrado no Pré-enchimento 0,32 $\pm 0,14$ $\mathrm{kg} / 1000 \mathrm{~m}^{2}$ de redes*16h, enquanto o maior foi registrado no Pós II 3,14 $\pm 0,46 \mathrm{~kg} / 1000 \mathrm{~m}^{2}$ de redes*16h (Tabela 1).

Tabela 1. Resultado da análise de variância unifatorial aplicada aos dados transformados de captura por unidade de esforço CPUEn (indivíduos $/ 1000 \mathrm{~m}^{2}$ rede*16hs) e CPUEb (kg/1000 m² rede*16hs), em função dos períodos e sítios de amostragem.

\begin{tabular}{lcccc}
\hline CPUE & Pré & Pós I & Pós II & Valor-p \\
\hline & & Montante & \\
CPUEn & $20,72 \pm 3,30 \mathrm{a}$ & $11,75 \pm 2,08 \mathrm{a}$ & $19,65 \pm 2,77 \mathrm{a}$ & 0,057 \\
CPUEb & $3,17 \pm 0,57 \mathrm{a}$ & $2,00 \pm 0,33 \mathrm{a}$ & $3,48 \pm 0,45 \mathrm{a}$ & 0,074 \\
& & Reservatório & 0,014 \\
CPUEn & $15,77 \pm 1,70 \mathrm{a}$ & $21,39 \pm 4,47 \mathrm{a}$ & $29,38 \pm 2,47 \mathrm{~b}$ & 0,011 \\
CPUEb & $2,23 \pm 0,25 \mathrm{a}$ & $2,07 \pm 0,32 \mathrm{a}$ & $3,19 \pm 0,22 \mathrm{~b}$ & \\
& & Jusante & 0,001 \\
CPUEn & $2,37 \pm 1,06 \mathrm{a}$ & $17,39 \pm 3,53 \mathrm{~b}$ & $17,16 \pm 2,40 \mathrm{~b}$ & 0,001 \\
\hline
\end{tabular}

*Letras distintas na mesma linha e cor vermelha indicam diferenças significativas $(\mathrm{p}<0,05)$. Fonte: Dados oriundos da pesquisa.

Comparando-se as três fases, no montante, verificou-se pouca alteração na estrutura em tamanho, sendo que na fase Pré a maior frequência foi de indivíduos na classe de comprimento de 12,0 a 12,9 cm (30\%), verifica-se também que 78,2\% dos indivíduos apresentam comprimento entre 9,0 a 12,9 cm. Na fase Pós I a frequência foi mais equitativa entre as classes, destacando-se a classe de 13,0-13,9 cm (18,7\%), enquanto que 68,0\% dos indivíduos capturados encontravam-se em comprimentos de 10,0 a 13,9 cm. Já na fase Pós II, a classe que mais se destacou foi de 11,0 a 11,9 cm (27\%), sendo que 81\% dos indivíduos capturados encontravam-se entre 10,0 a 13,9 cm (Figura 3A).

No reservatório foi observada uma alteração maior na estrutura em tamanho, quando comparadas as fases, sendo que na fase Pré a frequência foi mais distribuída entre as classes, com maior abundância para a classe de 10 a $10,9 \mathrm{~cm}(19,5 \%)$. Na fase Pós I, o tamanho médio foi o menor entre as fases, sendo que 36,6\% dos indivíduos estiveram na classe de 7,0 a 7,9 cm, enquanto que na fase Pós II, 40,8\% dos indivíduos capturados estiveram na classe 9,0 a 9,9 cm (Figura 3B).Também na jusante, se percebe uma alteração na estrutura em comprimento, porém diferente daquela observada no reservatório. Na fase Pré a maior frequência de indivíduos foi registrada para a classe de 10,0 a 10,9 cm (42,2\%), na fase Pós I o predomínio também foi nesta classe, porém com uma frequência menor (21,8\%). Já na fase Pós II, percebe-se um aumento no tamanho médio com 30,6\% dos indivíduos pertencentes à classe de 13,0 a 13,9 cm (Figura 3C). 
Figura 3. Porcentagem de fêmeas de Psalidodon aff. fasciatus de dezembro de 2007 a setembro de 2016, agrupados por classes de comprimento total (cm) para as três áreas de influência da PCH São Francisco Verdadeiro. A - MON; B - RES; C JUS.

A

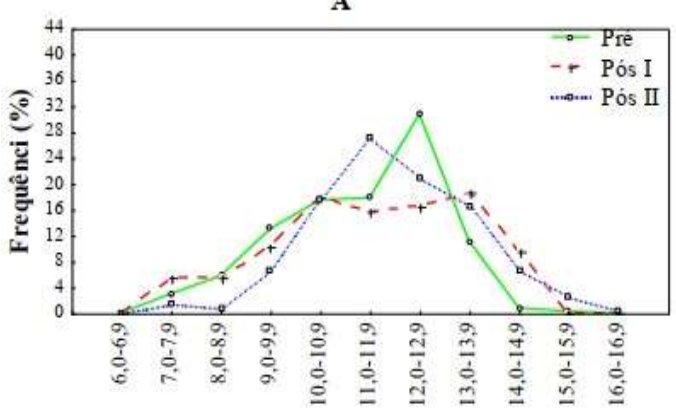

Classes de Comprimento (cm)

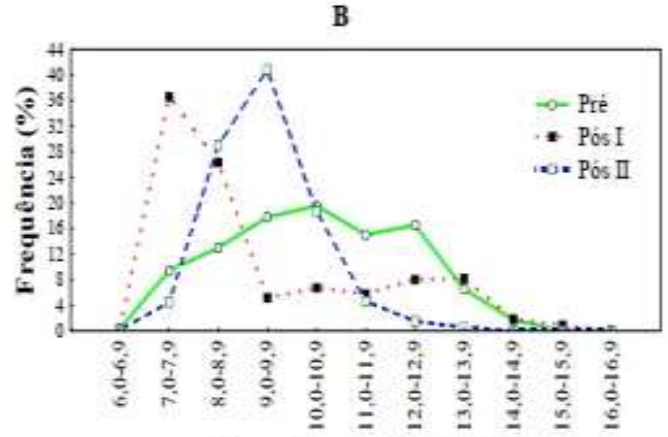

Classse de comprimento $(\mathrm{cm})$

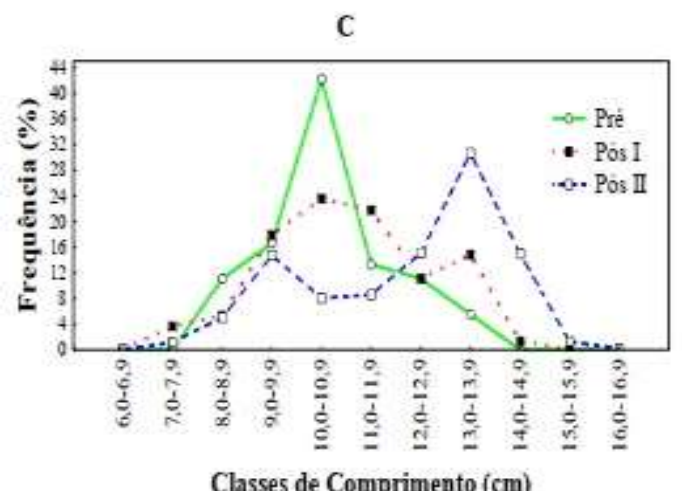

Fonte: Dados oriundos da pesquisa.

Com relação ao índice de atividade reprodutiva (IAR) no ambiente montante, não foi verificada diferença significativa entre as fases ( $\mathrm{p}>0,05)$, porém é possível verificar que houve um aumento na atividade após o barramento. Nas fases Pré e Pós II a atividade reprodutiva foi considerada intensa (14,34 e 18,82 respectivamente), enquanto que na fase Pós I ela foi considerada muito intensa $(20,69)$ (Figura 4A). O comportamento inverso pode ser observado para o reservatório onde no período Pré-enchimento a atividade reprodutiva foi considerada como intensa $(17,85)$, enquanto na fase Pós I a atividade foi classificada como moderada $(9,67)$ e no período Pós II ela foi classificada como muito intensa $(20,36)$. Percebe-se que a atividade reprodutiva no período Pós I diferiu significativamente dos outros períodos (p<0,05) (Figura 4B). Porém, no ambiente a jusante, a atividade reprodutiva ao longo do tempo variou de muito intensa na fase Pré $(33,54)$, a intensa na fase Pós I $(18,47)$ e na fase Pós II a atividade reprodutiva foi considerada como muito intensa $(20,10)$, entretanto, não houve diferença significativa ( $p>0,05)$ (Figura $4 \mathrm{C})$. 
Figura 4. Média e erro padrão do Índice de Atividade Reprodutiva de fêmeas de Psalidodon aff. fasciatus agrupados por períodos de dezembro de 2007 a setembro de 2016, para as três áreas de influência da PCH São Francisco Verdadeiro. A MON; B - RES; C - JUS.

A

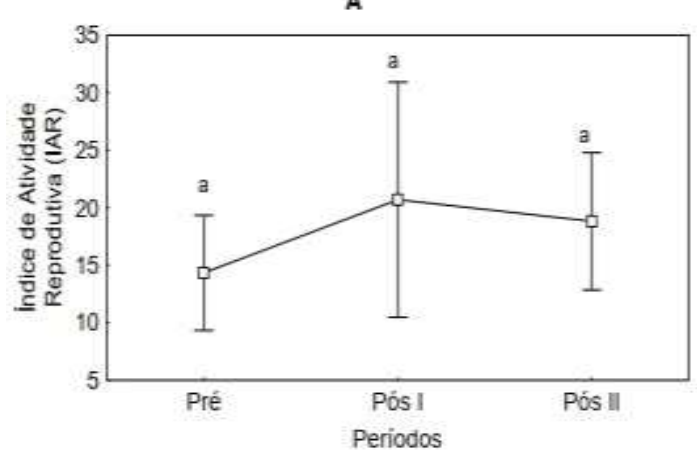

B

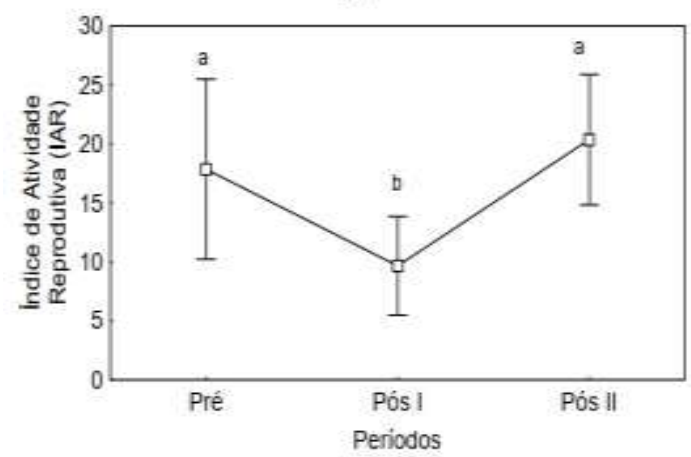

c

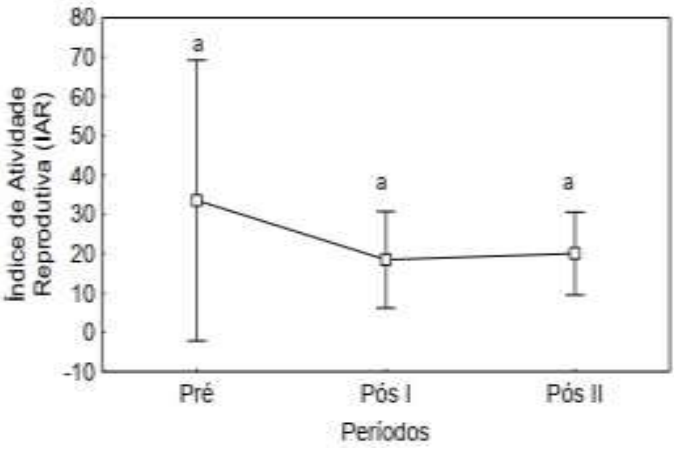

Fonte: Dados oriundos da pesquisa.

Quanto ao fator de condição, no montante, foi verificada diferença significativa entre as fases $(\mathrm{p}<0,05)$, sendo possível verificar uma diminuição do fator de condição com o passar dos períodos. O maior valor observado $(0,027)$ em Pré e o menor valor $(0,017)$ em Pós II (Figura 5A). No local onde foi formado o reservatório também foram verificadas diferenças significativas entre as fases $(\mathrm{p}<0,05)$, no primeiro período após a formação do reservatório houve um aumento no valor do

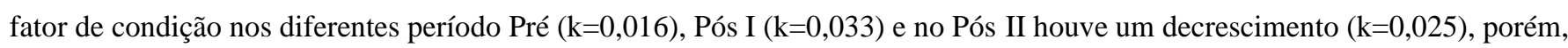
não sendo próximo ao observado antes da formação do reservatório (Figura 5B). Na jusante, também houve diferença significativa entre as fases $(\mathrm{p}<0,05)$, pois, antes da formação do reservatório os indivíduos possuíam higidez superior à encontrada após a formação do reservatório. Sendo que no período Pré apresentou k=0,086, Pós I k=0,020 e no período Pós II $\mathrm{k}=0,025$ (Figura 5C). 
Figura 5. Média e erro padrão do fator de condição de fêmeas de Psalidodon aff. fasciatus agrupados por períodos de dezembro de 2007 a setembro de 2016, para as três áreas de influência da PCH São Francisco Verdadeiro. A - MON; B - RES; C - JUS.
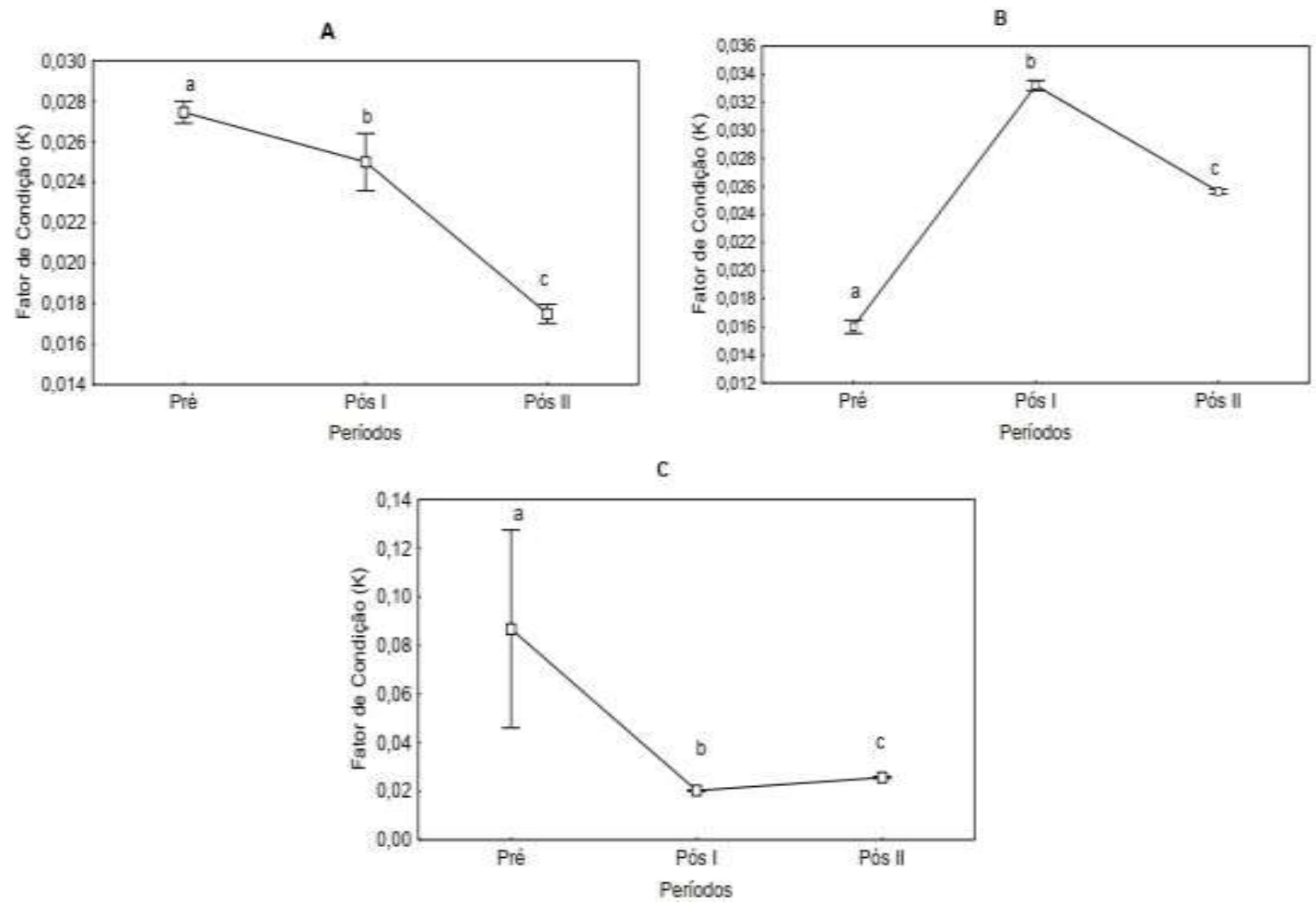

Fonte: Dados oriundos da pesquisa.

\section{Discussão}

Neste trabalho foram observados diferença significativa nas CPUEn e CPUEb para P. aff. fasciatus capturados a montante do reservatório da PCH São Francisco, indica que a interrupção do rio São Francisco Verdadeiro pela implantação do reservatório não afetou a abundância desta espécie. Alguns autores relatam pequenas alterações em locais a montante de barragem (Agostinho et al., 2007), e isto foi observado por Araújo et al. (2013) que avaliaram os períodos pré e pósenchimento do reservatório de Lajeado, no Tocantins, Brasil, e verificaram que no trecho a montante houve pouca variação na estrutura das espécies de peixes.

A manutenção do fluxo de água constante a montante de reservatórios de hidrelétricas, segundo Agostinho et al. (2016) é o que garante condições de alimentação e reprodução para as espécies reofílicas. E essas condições em ambiente represados favorece para abundância de P. aff. fasciatus após o enchimento do reservatório (Bennemann et al., 2005). Por outro lado, variações significativas com aumento da abundância na área represada foram registradas nos reservatórios das hidrelétricas de Rosana, Três Irmãos, Jordão e Corumbá (Agostinho et al., 1999; 2007; 2008; 2016). O aumento da abundância na área represada segundo Agostinho et al. (2016), se deve à transformação do ambiente lótico em lêntico passando a ser um ambiente com característica de águas paradas sem corrente, que aumenta a disponibilidade de recursos alimentares devido ao aumento da disponibilidade de nutrientes e consequentemente ao aumento da produtividade primária (Agostinho et al. 2007). O mesmo foi observado por Hahn et al. (2004) em estudo com espécies forrageiras que apresentaram aumento da plasticidade alimentar. Deste modo, ficou evidente que o aumento da abundância da espécie $P$. aff. fasciatus foi devido a disponibilidade de recursos alimentares após a formação do reservatório (Novakowski et al., 2007).

As alterações na abundância de espécies a jusante de pequena central hidrelétrica, praticamente não são relatadas na literatura, uma vez que estudos de pequenas barragens são escassos, principalmente com espécies não migradoras. 
Possivelmente, a elevação da abundância de $P$. aff. fasciatus a jusante da barragem esteja relacionada com a operação da usina, já que o fluxo de água é variável e dependente da geração de energia, o que estaria simulando períodos de seca e cheia que naturalmente ocorriam na região. Como a operação da PCH São Francisco é contínua e as variações de fluxo são em números muito maiores do que aconteciam anteriormente no curso do rio, a espécie recebeu mais estímulos para se reproduzir, aumentando significativamente as capturas por unidade de esforço em número e biomassa.

Em relação à estrutura em tamanho, pouca variação foi verificada a montante, sendo que a frequência de tamanho foi mais homogeneamente distribuída entre as classes, o que se deve à manutenção das características naturais do rio antes do represamento. Já na área represada, percebeu claramente que após o represamento os tamanhos médios foram menores e mais frequentes no Pós I e Pós II. Este resultado aponta que houve uma maior ocupação por indivíduos de menor porte, ou seja, o recrutamento foi maior no período pós enchimento. De acordo com Agostinho et al. (2007; 2016) existe uma tendência de ocupação de reservatórios por indivíduos menores, principalmente de espécies oportunistas como $P$. aff. fasciatus, que aproveita a disponibilidade de novos habitats e recursos alimentares (Novakowski et al., 2007, Morais et al., 2020).

As variações do reservatório e a jusante foram semelhantes para espécie $P$. aff. fasciatus, sendo que, após a formação do reservatório, foi registrada maior presença de indivíduos em classes de comprimento menores. Apesar deste comportamento ser similar no reservatório e jusante, a espécie não se adaptou as mudanças dos novos habitats. Essa alteração pode estar associada principalmente à variação de fluxo de água e também devido ao alimento exportado pelo reservatório.

De acordo com os resultados deste trabalho, ficou evidente que houve atividade reprodutiva durante todo o período de estudo, e, em todos os locais amostrados. No entanto, montante e jusante houve variação na intensidade reprodutiva, porém, não foram significativas. Evidenciando que nesses locais, a implantação da PCH São Francisco não influenciou na intensidade da atividade reprodutiva. Por outro lado, no reservatório, houve diferenças significativas no índice de atividade reprodutiva, sendo que, no período Pós I teve baixo índice e nos períodos Pré e Pós II teve um alto índice. Este resultado evidencia que, no primeiro período após o enchimento do reservatório, houve uma redução na intensidade reprodutiva na área alagada. A explicação mais plausível para este fato, segundo Suzuki et al. (1997) e Baumgartner et al. (2016) é que as espécies do gênero Astyanax, apresentaram maior índice de atividade reprodutiva a jusante da Barragem das usinas de Salto Segredo e Salto Santiago, respectivamente, indicando que elas preferem ambientes com características lóticas para a reprodução (Silva et al., 2010), deste modo, no caso de P. aff. fasciatus na PCH São Francisco, a desova ocorreu a montante e a prole derivou para dentro do reservatório, onde encontrou condições adequadas para o seu desenvolvimento, o que determinou um aumento da abundância neste local após o barramento.

A redução do grau de higidez das fêmeas a montante do reservatório após o represamento pode estar relacionado as mudanças do ambiente (Agostinho et al., 2007), favorecendo aos indivíduos condições para o crescimento, mas ao mesmo tempo não favoreceu a reprodução dos indivíduos, já que neste local as condições de fluxo de água foram mantidas.

Neste trabalho, foram observados que no reservatório o fator de condição aumentou no período Pós I, o que está relacionado ao aumento de nutrientes disponíveis com a formação do reservatório e contribuindo para as características do $P$. aff. fasciatus que possui uma alta plasticidade alimentar. Além disso, Lizama e Ambrósio (2002) citam que elevados valores de fator de condição, são encontrados para peixes que estão em fase de crescimento. Por outro lado, Agostinho et al. (2007) citam uma tendência decrescente na concentração de nutrientes culminando na redução da produtividade primária com o passar do tempo, o que poderia explicar a redução do fator de condição no período Pós II. Porém, devido ao baixo número de indivíduos capturados na jusante, o resultado de atividade reprodutiva apresentou-se elevado e podendo está relacionado as condições favoráveis do ambiente (Silva et al., 2010).

\section{Conclusão}


Os resultados nos permitiram concluir que a implantação da PCH São Francisco influenciou positivamente na abundância da espécie $P$. aff. fasciatus nos locais reservatório e jusante, sendo a primeira hipótese confirmada. Em relação à estrutura em tamanho corporal, verificou-se também que houve influência positiva a jusante e no reservatório, de modo que nestes ambientes foram ocupados principalmente por indivíduos de menor porte e aumentaram o tamanho médio da população com o passar do tempo, evidenciando que a segunda hipótese também foi confirmada. Por outro lado, a terceira hipótese foi rejeitada, já que nos locais jusante e montante, a atividade reprodutiva não apresentou alteração significativa, e no reservatório logo após a formação do reservatório houve uma redução da intensidade da reprodução. A quarta hipótese foi confirmada, apesar que a montante do reservatório o fator de condição diminuiu para a espécie, mas na jusante e reservatório houve influência positiva. Neste contexto, sugerimos mais estudos relacionados ao represamento por pequenas centrais hidrelétricas e soluções para diminuir os impactos ou restaurar a biodiversidade após a construção.

\section{Agradecimentos}

Os autores agradecem à Universidade Estadual do Oeste do Paraná (UNIOESTE), Grupo de Pesquisas em Recursos Pesqueiros e Limnologia (GERPEL) e ao corpo técnico do Instituto Neotropical de Pesquisas Ambientais (INEO) por suas contribuições para o desenvolvimento deste estudo. O agradecimento se estende à CAPES pela cessão da bolsa de estudos na modalidade Demanda Social.

\section{Referências}

Affonso, I. P., Azevedo, R. F., Santos, N. L. C., Dias, R. M., Agostinho, A. A. \& Gomes, L. C. (2015). Pulling the plug: strategies to preclude expansion of dams in Brazilian rivers with high priority for conservation. Natureza \& Conservação, 13(2): 199-203.

Agostinho, A. A., Gomes, L. C, Santos, N. C. L., Ortega, J. C. G. \& Pelicice, F. M. (2016). Fish assemblages in Neotropical reservoirs: Colonization patterns, impacts and management. Fisheries Research, 173: 26-36.

Agostinho, A. A., Gomes, L. C. \& Pelicice, F. M. (2007). Ecologia e manejo de recursos pesqueiros em reservatórios do Brasil. Eduem.

Agostinho, A. A., Miranda, L. E., Bini, L. M., Gomes, L. C., Thomaz, S. M. \& Suzuki, H. I. (1999). Patterns of colonization in Neotropical reservoirs, and prognoses on aging. Tundisi, J. G. \& Straskraba, M. eds. Theoretical reservoir ecology and its applications. Leiden, International Institute of Ecology.

Agostinho, A. A., Pelicice, F. M. \& Gomes, L. C. (2008). Dams and the fish fauna of the neotropical region: impacts and management related to diversity and fisheries. Brazilian journal of biology, 68: 1119-132.

Araújo, E. S., Marques, E. E., Freitas, I. S., Neuberger, A. L., Fernandes, R. \& Pelicice, F. M. (2013). Changes in distance decay relationships after river regulation: similarity among fish assemblages in a large Amazonian river. Ecology of Freshwater Fish, 22(4): 543-552.

Baumgartner, M. T., Silva, P. R. L. \& Baumgartner, G. (2016). Population structure and reproductive biology of Astyanax gymnodontus (characiformes: characidae), a poorly known endemic fish of the Iguaçu River basin, Brazil. Revista de biologia tropical 64(1): 69-77.

Bazzoli, N., Rizzo, E., Chiarini-Garcia, H. \& Ferreira, R. M. A. (1991). Ichthyofauna of the Paranaíba River in the area to be flooded by the Bocaina reservoir. Ciência e Cultura, 43(6): 451-53.

Bennemann, S. T., Gealh, A. M., Orsi, M. L. \& Souza, L. M. D. (2004). Ocorrência e ecologia trófica de quatro espécies de Astyanax (Characidae) em diferentes rios da bacia do rio Tibagi, Paraná, Brasil. Iheringia. Série Zoologia, 95: 247-254.

Cooper, A. R., Infante, D. M., Wehrly, K. E., Wang, L. \& Brenden, T. O. (2016). Identifying indicators and quantifying large- scale effects of dams on fishes. Ecological Indicators, 61: 646-657.

Ferreira, R. S.(2020). Os efeitos negativos causados pela construção e operação de usinas hidrelétricas. Research, Society and Development, 9(7): e543972406-e543972406.

Graça, W. J. \& Pavanelli, C. S. (2007). Peixes da planície de inundação do alto rio Paraná e áreas adjacentes. Eduem.

Gubiani, E. A., Daga, V. S., Frana, V. A., Graça, W. J. (2010). Fish, Toledo urban streams, São Francisco Verdadeiro River drainage, upper Paraná River basin, state of Paraná, Brazil. Check List, 6(1): 45-48.

Hahn, N. S., Fugi, R. \& Adrian, I. F. 2004. Trophic ecology of the fish assemblages. In: Thomaz, S. M., Agostinho, A. A.; Hahn, N. S. (Ed). The Upper Paraná River and its Floodplain: physical aspects, ecology and conservation. Leiden: Backhuys Publishers, 1: 247-69.

Holcomb, J. M., Nichols, R. B. \& Gangloff, M. M. (2016). Effects of small dam condition and drainage on stream fish community structure. Ecology of Freshwater Fish, 25(4): 553-564. 
King, M. (2005). Fisheries biology: assessment and management. Fishing News Books, Blackwell Science.

Lowe-McConnell, R. H. (1999). Estudos ecológicos de comunidades de peixes tropicais. In Estudos ecológicos de comunidades de peixes tropicais. 534-534 p.

Mérona, B. \& Albert, F. (1999). Ecological monitoring of fish assemblages downstream of a hydroelectric dam in French Guiana (South America). Regulated Rivers: Research \& Management: An International Journal Devoted to River Research and Management, 15(4): 339-351.

Morais, J. V. M., Pinheiro, S. C. C., Ahndrew, L. M. S., de Sousa, E. B. \& Paes, E. T. (2020). Composição e densidade do zooplâncton no reservatório da usina hidrelétrica de Tucuruí (Pará, Brasil). Research, Society and Development, 9(12): e9991210766-e9991210766.

Normando, F. T., Santiago, K. B., Gomes, M. V. T., Rizzo, E. \& Bazzoli, N. (2014). Impact of the Três Marias dam on the reprodution of the forage fish Astyanax bimaculatus and Astyanax fasciatus from the São Francisco River, Downstream from the dam, southeastern Brazil. Environmental biology of fishes. 97(3): 309-19.

Novaes, J. L. C., Moreira, S. I. L., Freire, C. E. C., Sousa, M. M. O. \& Costa, R. S. (2014). Fish assemblage in a semi-arid Neotropical reservoir: composition, structure and patterns of diversity and abundance. Brazilian Journal of Biology, 74(2): 290-301.

Novakowski, G. C., Hahn, N. S. \& Fugi, R. (2007). Alimentação de peixes piscívoros antes e após a formação do reservatório de Salto Caxias, Paraná, Brasil. Biota Neotropica, 7: 149-154.

Okamura, D., Araújo, F. G. D., Rosa, P. V., Freitas, R. T. F., Murgas, L. D. S. \& Cesar, M. P. (2010). Effect of benzocaine concentration and fish size on anesthesia and recovery in Nile tilapia. Revista Brasileira de Zootecnia. 39: 971-76.

Oliveira, A. G., Baumgartner, M. T., Gomes, L. C., Dias, R. M. \& Agostinho, A. A. (2018). Long-Term effects of flow regulation by dams simplify fish functional diversity. Freshwater Biology, 63(3): 293-305.

Poff, N. L. \& Schmidt J. C. (2016). How dams can go with the flow: Small changes to water flow regimes from dams can help to restore river ecosystems. Science, 353(6304): 1099-100.

Silva, J. P. A. D., Muelbert, A. E., Oliveira, E. C. D., \& Fávaro, L. F. (2010). Reproductive tactics used by the Lambari Astyanax aff. fasciatus in three water supply reservoirs in the same geographic region of the upper Iguaçu River. Neotropical Ichthyology, 8: 885-892.

Sokal, R. R. \& Rohlf, F. J. (1981). Congruência taxonômica em Leptopodomorpha reexaminada. Systematic Zoology, 30 (3), $309-325$.

Suzuki, H. I., Pavanelli, C. S., Fugi, R., Bini, L. M. \& Agostinho, A. A. (1997). Ictiofauna de quatro tributários do reservatório de Segredo. Reservatório de Segredo: bases ecológicas para o manejo. In: Agostinho A. A. \& Gomes L. C. (Eds.), EDUEM, 259-273.

Suzuki, H. I., Vazzoler, A. E. A. M., Marques, E. E., Lizama, M. P., Inada, P., Thomaz, S. M. \& Hahn, N. S. (2004). The upper Paraná River and its floodplain: physical aspects, ecology and conservation.

Terán, G. E., Benitez, M. F. \& Mirande, J. M. (2020). Opening the Trojan horse: phylogeny of Astyanax, two new genera and resurrection of Psalidodon (Teleostei: Characidae). Zoological Journal of the Linnean Society, 190(4), 1217-1234.

Thomaz, S. M., Agostinho, A. A. \& Hahn, N. S. (2004). The Upper Paraná River and its floodplain: physical aspects, ecology and conservation. (Eds.), Backhuys Publishers.

Vazzoler, A. E. A. M. (1981). Manual de métodos para estudos biológicos sobre populações de peixes: Crescimento e Reprodução. Brasília: CNPq, Programa nacional de zoologia.

Vazzoler, A. E. A. M. (1996). Biologia da Reprodução de peixes teleósteos: teoria e prática. Eduem, 169.

Viana, L. F., do Amaral Crispim, B., Barufatti, A. \& Lima-Junior, S. E. (2020). Existem diferenças na frequência de micronúcleos em Astyanax lacustris em relação ao sexo, massa e comprimento? Research, Society and Development, 9(8): e181985151.

Winemiller, K. O. (1989). Patterns of variation in life history among South American fishes in seasonal environments. Acta Oecologia, 81(2): 225-241.

Zar, J. H. (1999). Biostatistical analysis. Pearson Education India. 